\title{
Detektion von Kunststoffen in Wasser mithilfe von Fluoreszenz
}

\author{
Maximilian Wohlschläger ${ }^{1}$, Martin Versen ${ }^{1}$ \\ ${ }^{1}$ Technische Hochschule Rosenheim, Hochschulstraße 1, 83024 Rosenheim, Deutschland
}

\begin{abstract}
Zusammenfassung
Die wohl größte aktuelle Umweltverschmutzung mit Kunststoffen findet in Ozeanen, Seen und Flüssen statt. Bis jetzt gibt es kein etabliertes Verfahren, mit dem Kunststoffe in Umwelt- bzw. Wassermatrizen detektiert werden können. Deshalb wird mit einem Verfahren, welches auf dem Prinzip der Fluoreszenz basiert, untersucht, ob unter Laborbedingungen Kunststoffe in Wasser detektiert und identifiziert werden können. Anhand eines mathematischen Modells, einer Simulation und mit Hilfe von optischen Experimenten wird gezeigt, dass der Nachweis und auch eine Identifikation von Kunststoffen in Abhängigkeit der Wassertiefe, mit diesem Verfahren möglich ist.
\end{abstract}

Keywords: Kunststoffe, Umwelt, Fluoreszenzmesstechnik, Fluoreszenz

\section{Einleitung}

Weltweit sind Kunststoffe mittlerweile in allen Bereichen der Industrie als Aufbewahrungs-, Schutz- und Verpackungswerkstoff unverzichtbar. Gleichzeitig steigt die Anzahl verschiedener Kunststoffsorten stetig an und die Verfahren zum Kunststoffrecycling werden immer weiter verbessert, um eine möglichst hohe Qualität bei der Wiederverwendung zu gewährleisten.

Kunststoffe können jedoch nur dann wiederverwendet werden, wenn diese ordnungsgemäß entsorgt werden. Leider ist dies aber aufgrund von z.B. mangelnder Aufklärung oder fehlenden Müllentsorgungssystemen nicht immer der Fall. Daher wächst die Umweltbelastung derzeit ins Unermessliche.

So zeigt beispielsweise eine Studie aus Hongkong, dass 27.909 Kunststoffpartikel in einem Wasservolumen von $100 \mathrm{~m}^{3}$ in Küstenregionen vor Hongkong zu finden sind. Betrachtet man Studien aus Neuseeland in der Küstenregion von Queen Charlotte Sound, erscheinen die Werte aus Hongkong harmlos, denn dort wurden 763.000 Partikel in $100 \mathrm{~m}^{3}$ Wasser festgestellt. In Ostchina wurden wiederrum nur 17 Partikel pro $100 \mathrm{~m}^{3}$ und im Küstenraum von Portugal 4 Partikel pro $100 \mathrm{~m}^{3}$ Wasser nachgewiesen (siehe [1] und die darin enthaltenen Referenzen).

Die Verschmutzung der Umwelt ist allerdings nur ein Aspekt dieses Problems, denn Meereslebewesen wie Fische nehmen die im Wasser enthaltenen Partikel auf, wie Studien malaysischer und kanadischer Forscher zeigen $[2,3]$. Durch den Verzehr der kontaminierten Fische kann dies auch zum Gesundheitsrisiko für den Menschen führen.

Zum Nachweis der Kunststoffe in Wasser und zur Analyse werden die Kunststoffe aus dem Wasser manuell ausgesiebt, ins Labor transportiert und dort mit spektrometrischen Verfahren wie zum Beispiel der Infrarot oder Raman Spektroskopie untersucht [4]. Es gibt keine Methode, welche den Kunststoff vor Ort in einer Wassermatrix detektieren und identifizieren kann. Um diese Lücke zu schließen, wird ein optisches Verfahren gesucht, dass Kunststoff in Wasser erkennt und identifiziert.

Derzeit gibt es zwei unter Laborbedingungen getestete Verfahren, die Kunststoffe detektieren und voneinander unterscheiden können.

Eines davon ist die Trennung der Kunststoffe aufgrund inrer spezifischen Fluoreszenzabklingzeiten. Eine Probe wird dabei mit einem Laserlichtimpuls angeregt und es wird die Zeit gemessen, wie lange der Kunststoff nachleuchtet. Die sich daraus ergebende Fluoreszenzabklingzeit ist für jeden Kunststoff spezifisch und lässt eine Unterscheidung zu [5, 6]. Da die Abklingzeiten in der Größenordnung von Nanosekunden liegen, sind für die Realisierung eines Messaufbaus synchronisationsschnelle und folglich teure Geräte erforderlich.

Ein zweites Verfahren betrachtet die spezifische Fluoreszenz-Quanteneffizienz. Dabei wird der Kunststoff mithilfe einer 
Lichtquelle angeregt und das Fluoreszenzsignal gemessen. Durch spektrale Rechenoperationen kann nach der Messung die Anzahl der Fluoreszenz-Photonen berechnet werden. Ist das Absorptionsspektrum des Kunststoffes bekannt, kann die Anzahl der absorbierten Photonen ermittelt werden. Die spezifische Quanteneffizienz wird dann berechnet aus dem Verhältnis der Anzahl an Fluoreszenz-Photonen zur Anzahl der absorbierten Photonen. Darüber hinaus kann bei diesem Verfahren eine kunststoffspezifische Apparatekonstante gebildet werden, die definiert ist als das Verhältnis aus der Anzahl der von der Detektionseinheit gezählten Photonen zur Anzahl der absorbierten Photonen [7, 8].

Bei beiden Verfahren werden Anregungswellenlängen im nahen UVIVIS-Bereich (ultravioletter visueller- Bereich) des elektromagnetischen Spektrums eingesetzt. Vorteilhaft ist, dass Licht in diesem Wellenlängenbereich eine hohe Eindringtiefe in Wasser hat, wie Abb. 1 zeigt (siehe Wellenlängenbereich von 380-400nm).

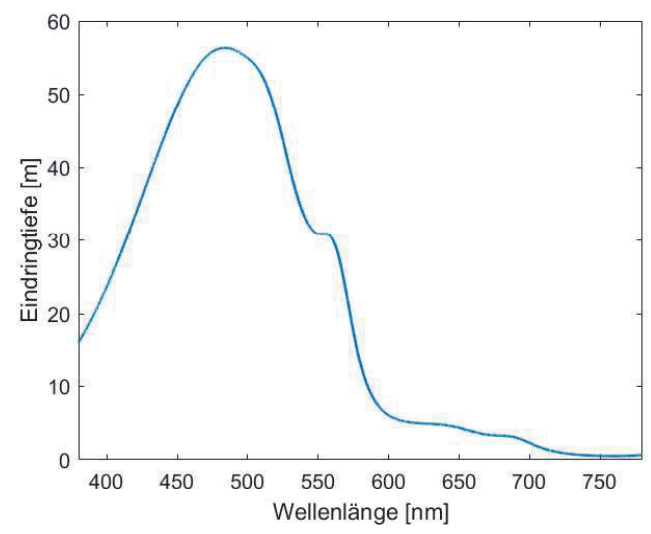

Abb. 1: Darstellung der Lichteidringtiefe in Wasser, in Abhängigkeit der Wellenlänge, berechnet mit dem Absorptionskoeffizienten aus [9].

Zur Berechnung wird der Absorptionskoeffizienten von reinem Wasser aus [9] verwendet, welcher keine Schwebepartikel, Verunreinigungen etc. berücksichtigt.

Im Folgenden wird untersucht, ob Kunststoffe aufgrund ihrer spezifischen Quanteneffizienz bzw. Apparatekonstante in Wasser detektiert und identifiziert werden können. Mit einer Simulation wird die Wassertiefe bestimmt, bis zu der ein Kunststoff auffindbar ist. Darüber hinaus soll überprüft werden, ob ein Zusammenhang aus der Theorie und Realität hergestellt werden kann.

Der Beitrag gliedert sich in die Weiterentwicklung und Modifikation des theoretischen und heuristischen Modells aus [7, 8], dem Simulationsmodell und den Simulations- ergebnissen, sowie den experimentellen Untersuchungen und den daraus resultierenden Ergebnissen. Am Ende wird eine Zusammenfassung einen Überblick über die Ergebnisse und den Zusammenhang von Theorie und Realität geben.

\section{Theoretisches Modell zur Detektion des Kunststoffes in Wasser}

In diesem Kapitel wird in den Stand der Technik eingeführt und die Modifikation des theoretischen Modells erläutert.

\subsection{Stand der Technik}

Das Modell zur Detektion und Identifikation von Kunststoffen wurde bereits in [7, 8] vorgestellt (siehe modifiziertes Modell Abb. 2). Ein Lichtstrahl trifft mit einer definierten Anzahl an Photonen (2) auf die Kunststoffprobe (3). Die Absorption eines Photons hat zur Folge, dass ein Elektron von einem niedrigen Energieniveau auf ein höheres Energieniveau gehoben wird. Da das Elektron bestrebt ist, in seinen energetischen Grundzustand zurückzukehren, gibt es seine Energie einerseits als nicht strahlende Energie und andererseits als strahlende Energie, einem Fluoreszenz-Photon, ab. Durch den Energieverlust, ist das Fluoreszenzphoton niederenergetischer und hat eine höhere Wellenlänge. Diese Wellenlängenverschiebung zwischen Anregungs- und Fluoreszenzlicht wird als Stokes-Verschiebung bezeichnet.

Die anregende Lichtquelle (1) ist dabei eine UV-LED mit einer CW (Zentralen Wellenlänge) von $390 \mathrm{~nm}$. Die exakte Strahlführung für (2), (4) und (5) wird mit einem Mikroskop gewährleistet. Als Detektionseinheit dient ein Minispektrometer. Mit diesem werden wellenlängenabhängige Aussagen getroffen, wie viele Photonen abhängig von der Wellenlänge detektiert werden.

Die Berechnung der Quanteneffizienz von den drei Kunststoffproben ist in [8] gezeigt und führt unter Berücksichtigung der Gaußschen Normalverteilung $\mathrm{zu}$ den in Tab. 1 eingetragenen Ergebnissen.

Tab. 1: Berechnete Werte der Quanteneffizienz QE und Apparatekonstante $Q E_{A}$ für die Kunststoffe $P A$ (Polyamid), PE (Polyethylen) und PET (Polyethylenterephthalat) [8]

\begin{tabular}{|c|c|c|}
\hline Polymer & QE $[\%]$ & $Q_{A}$ \\
\hline PA & $2,3 \pm 0,1$ & $2,2 \cdot 10^{-8} \pm 1 \cdot 10^{-9}$ \\
\hline PE & $3,0 \pm 0,2$ & $2,9 \cdot 10^{-8} \pm 1,9 \cdot 10^{-9}$ \\
\hline PET & $3,4 \pm 0,2$ & $3,4 \cdot 10^{-8} \pm 1,9 \cdot 10^{-9}$ \\
\hline
\end{tabular}




\subsection{Theoretisches Modell}

Um zu untersuchen, ob eine Detektion und Identifikation im Wasser möglich ist, wird das aus [8] bekannte Modell modifiziert und erweitert (siehe Abb. 2). Ein entscheidender Faktor für die Detektions- bzw. Identifikationsgrenze ist die Wasserschicht (7) die sich auf der Kunststoffprobe (3) befindet.

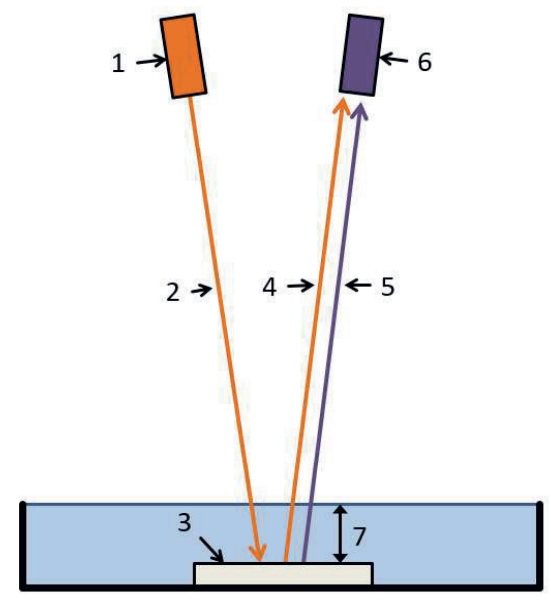

Abb. 2: Theoretisches Modell zur Detektion und Identifikation in Wasser mit Lichtquelle1, dem anregenden Lichtstrahl 2, der Kunststoffprobe 3, dem reflektierten Lichtanteil 4, dem fluoreszierenden Lichtanteil 5, der Detektionseinheit 6 und der Wasserhöhe über der Kunststoffprobe 7.

Um die exponentielle Absorption von Wasser zu berücksichtigen, wird der Absorptionskoeffizient von Waser nach [9] in das theoretische Modell aus [8] aufgenommen. Dabei ergibt sich die absorbierte Photonenanzahl $N_{A}$ nach GI. (1):

$N_{A}=N_{E} \cdot\left(1-e^{-\alpha_{P} \cdot d_{P}}\right) \cdot e^{-\alpha_{W} \cdot d_{W}}$

wobei $N_{E}$ die auf den Kunststoff treffenden Photonen (in Abb. 2 siehe Nummer 2), $\alpha_{P}$ der Absorptionskoeffizient, $d_{P}$ die Dicke des Kunststoffes, $\alpha_{w}$ der Absorptionskoeffizient von Wasser und $d_{w}$ die Höhe der Wasserschicht auf der Kunststoffprobe sind.

Mit der Anzahl der absorbierten Photonen $N_{A}$ und der Quanteneffizienz $Q E$ kann dann die Anzahl der Fluoreszenz-Photonen $N_{F}$ nach $G$. (2) berechnet werden.

$N_{F}=N_{A} \cdot Q E$

Aus den Fluoreszenz-Photonen wird dann mit Gl. (3) die Anzahl der detektierten Photonen $N_{D}$ unter Verwendung des Transmissionsgrades des Mikroskops $T_{M}$ bestimmt.

$$
N_{D}=N_{F} \cdot e^{-\alpha_{W} \cdot d_{W}} \cdot T_{M}
$$

Die Anzahl der detektierten Photonen ist eine der beiden Größen, die Aufschluss über eine mögliche Detektierbarkeit und Identifizierbarkeit liefern soll. Als zweites kann mit ihr die kunststoffspezifische Apparatekonstante $Q E_{A}$ nach $\mathrm{Gl}$. (4) berechnet werden.

$$
Q E_{A}=N_{D} / N_{A}
$$

Beide Größen können in Abhängigkeit der Wasserschicht auf dem Kunststoff dargestellt werden, was nachfolgende Simulation zeigt.

\section{Simulation der Identifikation in Wasser}

Zur Simulation wird ein Programm in der Berechnungssoftware MATLAB verwendet. Im Folgenden werden die Funktionsweise des Programmes und die resultierenden Simulationsergebnisse beschrieben

\subsection{Simulationsmodell}

Das erstellte Simulationsprogramm, arbeitet nach dem in Abb. 3 gezeigtem Ablaufplan.

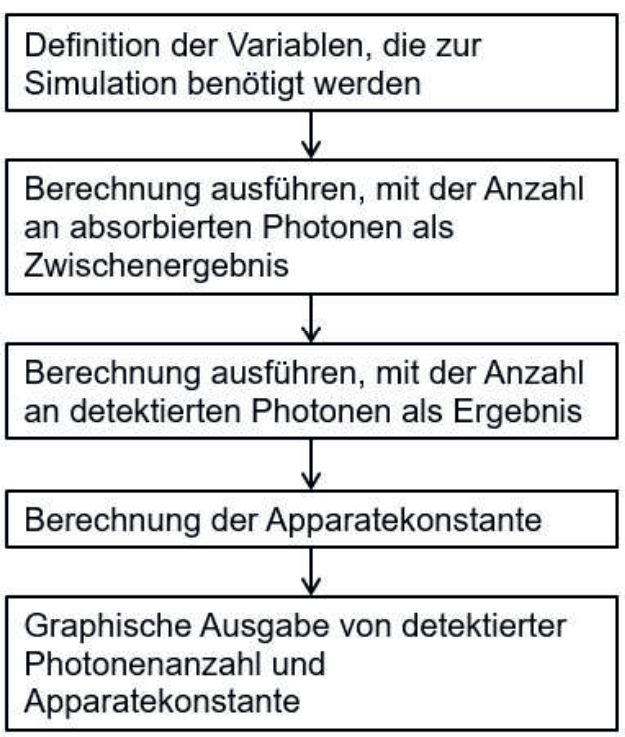

\section{Abb. 3: Programmablaufplan des zur Simulation verwendeten Berechnungsprogramms.}

Nachdem in einem ersten Schritt die für die Simulation benötigten Variablen definiert sind, wird die Berechnung der Anzahl der absorbierten Photonen ausgeführt und als Zwischenergebnis gespeichert. Mit dem Zwischenergebnis wird im vierten Schritt zusammen mit der Quanteneffizienz die Anzahl der Fluoreszenz-Photonen berechnet. Im dritten Schritt wird mit der Anzahl der FluoreszenzPhotonen die Anzahl der detektierten Photonen 
ermittelt. Zusätzlich wird aus der Anzahl der detektierten Photonen und der Anzahl der absorbierten Photonen die spezifische Apparatekonstante bestimmt.

Die berechneten Werte werden zugehörig zu ihrer Wasserschichtdicke in einer Matrix gespeichert, d.h. jeder Wasserhöhenwert hat einen zugehörigen Wert für die detektierte Photonenanzahl und Apparatekonstante.

Am Ende der Simulation werden zwei Diagramme ausgegeben, wobei eines davon die Anzahl der detektierten Photonen und das andere die Apparatekonstante als Funktion der Wasserhöhe auf dem Kunststoff zeigt.

Für den Graphen der Apparatekonstante wird in bestimmten Abständen der zugehörige Fehlerbalken eingetragen, der der Gauß'schen Standardabweichung der experimentell bestimmten Quanteneffizienz entspricht. Bei einer Überschneidung von zwei Fehlerbalken, ist es unwahrscheinlich, die Kunststoffe anhand ihrer spezifischen Apparatekonstante zuverlässig zu unterscheiden.

\subsection{Definition der Variablen}

Zur Simulation wurde die bereits in $[7,8]$ verwendeten Werte zur Berechnung der Quanteneffizienz und Apparatekonstante verwendet. Benötigt werden noch die in Tab. 1 eingetragenen Quanteneffizienzen der drei Kunststoffproben und die Wasserhöhe. Für diese wurde ein Wertebereich von $0 \mathrm{~mm}$ bis $50000 \mathrm{~mm}$ in $1,0 \mathrm{~mm}$ Schritten definiert.

\subsection{Simulationsergebnisse}

Die Ergebnisse der Simulation sind in Abb. 4 und Abb. 5 dargestellt, wobei Abb. 4 die Anzahl der detektierten Photonen Abb. 5 die Apparatekonstante als Funktion der Wasserschicht auf dem Kunststoff zeigt.

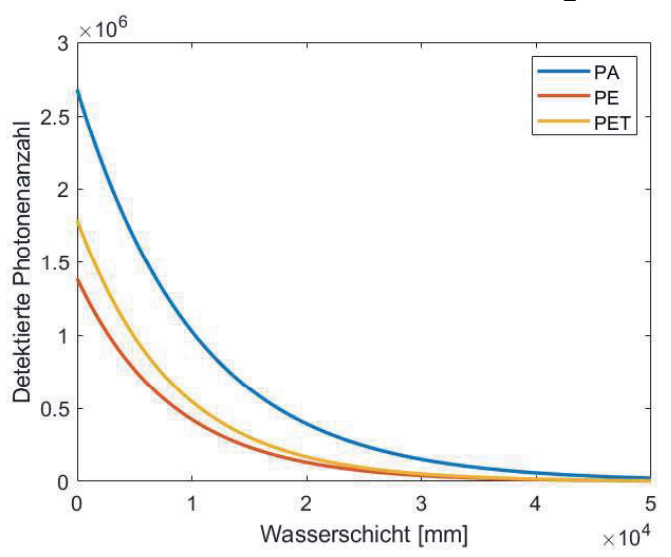

Abb. 4: Anzahl der detektierten Photonen in Abhängigkeit der Wasserhöhe, im Bereich von Omm bis 50000mm für die Kunststoffe PA, PE und PET

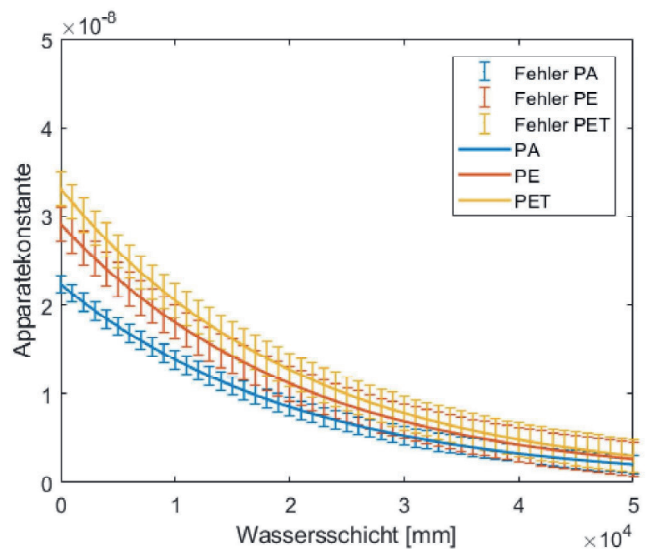

Abb. 5: Apparatekonstante mit Fehlerbalken in Abhängigkeit der Wasserhöhe, im Bereich von Omm bis $50000 \mathrm{~mm}$ für die Kunststoffe PA, PE und PET

Die Identifikation mit der Apparatekonstante ist allerdings bei $20 \mathrm{~m}$ nicht mehr möglich. Um eine simulative Aussage über die Identifizierbarkeit zu treffen, wird deshalb der Bereich zwischen Omm und $20000 \mathrm{~mm}(20 \mathrm{~m})$ vergrößert.

Die Vergrößerung ist in Abb. 6 dargestellt. Die Fehlerbalken von PA und PE überlappen bis zu einer Wassertiefe von ca. $18 \mathrm{~m}$ nicht. Eine Unterscheidung zwischen PA und PET ist sogar in tieferen Gewässern möglich. Folglich kann der Kunststoff PA mit hoher Wahrscheinlichkeit bis zur Wassertiefe von ungefähr $18 \mathrm{~m}$ als Polyamid identifiziert werden ohne mit PE oder PET verwechselt zu werden.

Zwischen den Kunststoffen PE und PET ist keine zuverlässige Unterscheidung bei über $2 \mathrm{~m}$ möglich, da dort eine Überschneidung der Fehlerbalken stattfindet. Folglich können diese zwei Kunststoffe nur bis zu einer Wassertiefe von kleiner $2 \mathrm{~m}$ relativ sicher identifiziert und unterschieden werden.

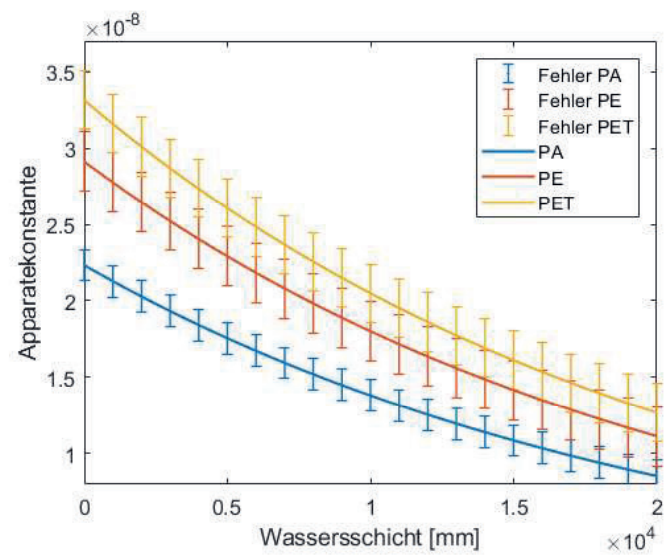

Abb. 6: Apparatekonstante mit Fehlerbalken in Abhängigkeit der Wasserhöhe, im Bereich von Omm bis 20000mm für die Kunststoffe PA, PE und PET 


\section{Experimente zur Detektion und Identifikation in Wasser}

Um das simulierte Ergebnis zu überprüfen, werden die Kunststoffe in Wasser präpariert und nach den in [7, 8] gezeigten Methoden ausgewertet. Im Folgenden wird die Probenvorbereitung, der für die Experimente verwendete Versuchsaufbau, das Vorgehen bei den Untersuchungen und die die daraus resultierenden Ergebnisse gezeigt.

\subsection{Probenvorbereitung}

Die in den Versuchen verwendeten Proben sind Folien der Kunststoffe PA, PE und PET, welche an der Technischen Hochschule Rosenheim hergestellt worden sind.

Die Folien werden in Stücken von $2 \mathrm{~cm} \times 2 \mathrm{~cm}$ in einer Petrischale mithilfe von Klebestreifen befestigt (siehe Abb. 7). Dadurch wird verhindert, dass die Kunststoffe aufschwimmen, da alle eine geringere Dichte als Wasser aufweisen.

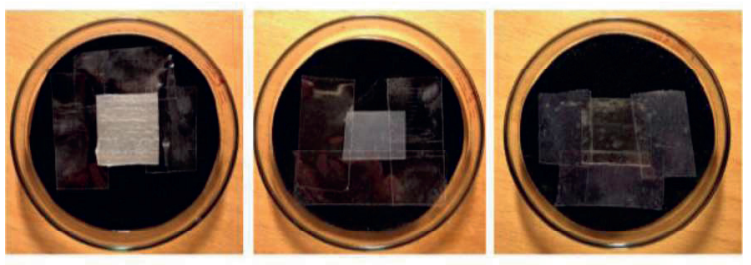

Abb. 7: Kunststoffproben PA (Links), PE (Mitte) und PET (Rechts) in einer Petrischale, mit Klebeband gegen Aufschwimmen befestigt.

\subsection{Experimenteller Aufbau}

Als experimenteller Aufbau wurde der in $[7,8]$ bereits erklärte Aufbau verwendet. Dieser besteht aus einer Lichtquelle einer ProbeStation und einem Minispektrometer.

Als Lichtquelle dient eine NVUS233B UV-LED von Nichia [10]. Wie bereits erwähnt, besitzt die LED eine zentrale Wellenlänge von $390 \mathrm{~nm}$, was optimal für die Anregung der Kunststoffproben ist.

An der Probe-Station EPS150FA von Cascade Microtech können die Komponenten, also Lichtquelle und Minispektrometer, befestigt werden. In der Probe-Station ist ein Mikroskop integriert, an welchem eine zwei, zehn oder zwanzigfache Vergrößerung eingestellt werden kann. Dies ist insbesondere vorteilhaft, um Photonen aus extrem kleinen Proben aufzuzeichnen [11].

Das Minispektrometer C10083CAH von Hamamatsu fungiert als spektrale Detektionseinheit. Es kann mit einer Auflösung von $1 \mathrm{~nm}$ den Wellenlängenbereich von 300 bis $1080 \mathrm{~nm}$ aufzeichnen [12].

\subsection{Vorgehen bei den Experimenten}

Bei den Experimenten wurde nach dem in Abb. 8 dargestellten Ablaufplan vorgegangen. Anzumerken ist, dass die Petrischale eine maximale Füllhöhe von $8 \mathrm{~mm}$ hat und daher keine Versuche in tieferem Wasser durchgeführt werden können.

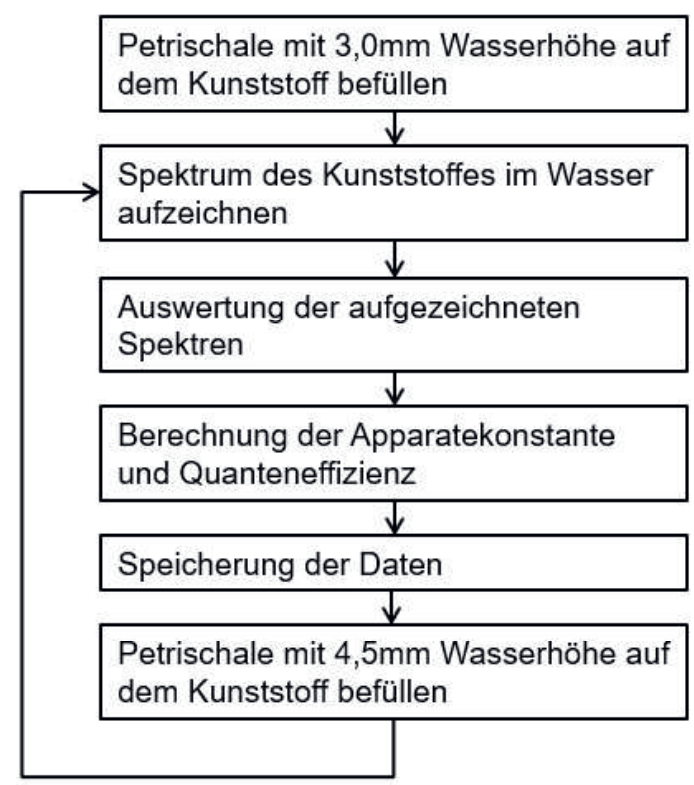

Abb. 8: Versuchsablaufplan zur Vermessung der Kunststoffproben in 3,0mm, 6,0mm und 8,0mm Wasser

Die Kunststoffproben sind im Trockenen und in den Wassertiefen von $3,0 \mathrm{~mm}, 4,5 \mathrm{~mm}, 6,0 \mathrm{~mm}$, $7,0 \mathrm{~mm}$ und $8,0 \mathrm{~mm}$ vermessen worden.

Die Versuche im Trockenen werden wie in [8] beschrieben durchgeführt und zeigen im Rahmen des Fehlers ähnliche Werte für die Quanteneffizienzen und Apparatekonstante. Zurückgeführt werden diese Änderungen auf statistische Einflüsse wie beispielsweise eine nicht kontrollierte Raumtemperatur bei den Versuchen. Es ergeben sich die in Tab. 2 eingetragenen Werte für die Quanteneffizienz und Apparatekonstante.

Tab. 2: Experimentell bestimmte Werte der Quanteneffizienz QE und Apparatekonstante $Q E_{A}$ für die Kunststoffe PA (Polyamid), PE (Polyethylen) und PET (Polyethylenterephthalat)

\begin{tabular}{|c|c|c|}
\hline & $Q E[\%]$ & $Q E_{A}$ \\
\hline PA & $1,97 \pm 0,08$ & $1,91 \cdot 10^{-8} \pm 8,16 \cdot 10^{-10}$ \\
\hline PE & $3,65 \pm 0,03$ & $3,52 \cdot 10^{-8} \pm 1,84 \cdot 10^{-9}$ \\
\hline PET & $4,36 \pm 0,03$ & $4,21 \cdot 10^{-8} \pm 2,99 \cdot 10^{-10}$ \\
\hline
\end{tabular}


Nach der Bestimmung der Quanteneffizienz im Trockenen, wird die Petrischale mit einem Wasserstand von $3,0 \mathrm{~mm}$ auf dem Kunststoff befüllt und unter das Mikroskop gestellt, um das Spektrum aufzunehmen. Die Kunststoffprobe wird an 20 unterschiedlichen Positionen vermessen. Die dabei aufgenommenen Spektren werden in Abhängigkeit der Wellenlänge in eine Tabelle übertragen und mit MATLAB ausgewertet. Anschließend werden die Mittelwerte und die Standardabweichungen unter Annahme einer Gauß'schen Normalverteilung berechnet.

Nach der Auswertung mit einer Wasserhöhe von $3,0 \mathrm{~mm}$ auf dem Kunststoff, wird die Petrischale weiter bis auf $4,5 \mathrm{~mm}$ mit Wasser befüllt. Anschließend wird der beschriebene Vorgang einschließlich der Auswertung der Daten wiederholt.

Wenn die Untersuchungen mit einer Wasserschicht von 4,5mm abgeschlossen sind, wird der Wasserstand weiter auf $6,0 \mathrm{~mm}, 7,0 \mathrm{~mm}$ und $8,0 \mathrm{~mm}$ erhöht und die Messungen werden wiederholt.

Wenn die erste Kunststoffprobe in den fünf Wassertiefen vermessen ist, wird der Versuch mit den anderen zwei Kunststoffproben nach dem gleichen Schema durchgeführt.

\subsection{Experimentelle Ergebnisse}

Die Auswertung der Daten zeigt, dass sich sowohl die Apparatekonstante als auch die Quanteneffizienz mit steigender Wasserhöhe ändert.

In Abb. 9 ist die Quanteneffizienz als Funktion der Wasserschicht für Messungen im Trockenen, bei $3,0 \mathrm{~mm}$, bei $4,5 \mathrm{~mm}$, bei $6,0 \mathrm{~mm}$, bei $7,0 \mathrm{~mm}$ und bei $8,0 \mathrm{~mm}$ Wasserhöhe auf dem Kunststoff dargestellt. Die Quanteneffizienz nimmt mit zunehmendem Wasserstand exponentiell ab.

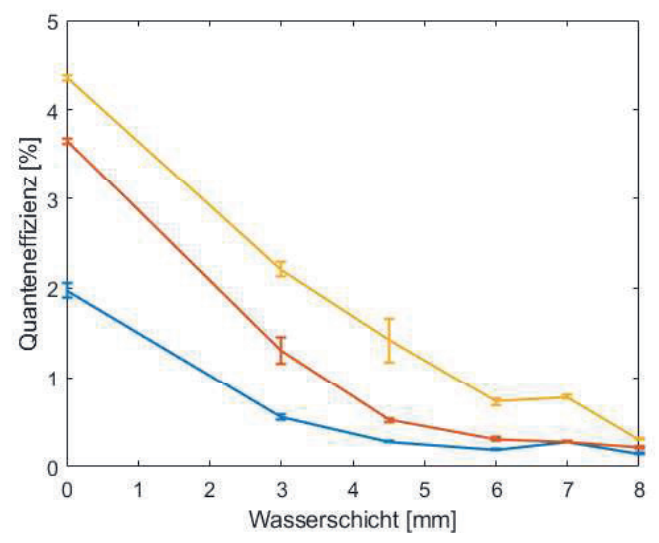

Abb. 9: Darstellung der Quanteneffizienz als Funktion der Wasserschicht für die drei verwendeten Kunststoffproben $P A, P E$ und $P E T$

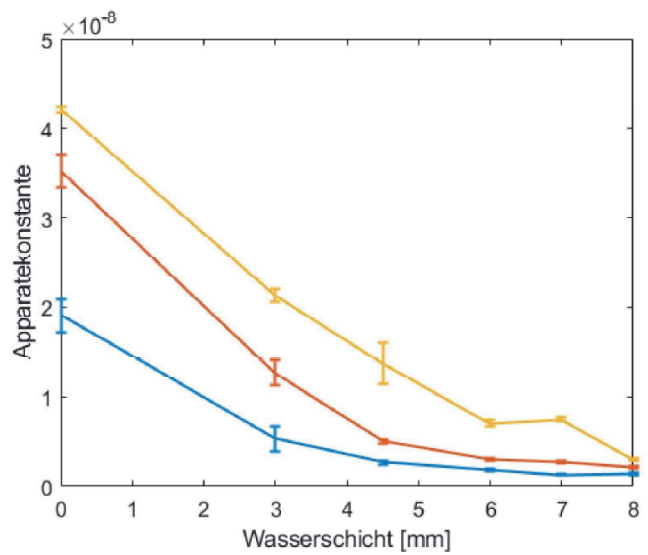

Abb. 10: Darstellung der Apparatekonstante in Abhängigkeit der Wasserschicht für die drei verwendeten Kunststoffproben PA, PE und PET

Für die Apparatekonstante in Abhängigkeit der Wasserschicht ergibt sich das in Abb. 10 gezeigte Diagramm.

Wie bei der Quanteneffizienz in Abb. 9 ist auch hier ein exponentieller Abfall zu sehen, welcher erwartet wird. Allerdings ist dieser deutlich höher, als erwartet, was im Folgenden näher betrachtet wird.

\subsection{Auswertung der experimentellen Ergebnisse}

Um eine Aussage über die experimentellen Ergebnisse zu treffen, werden Ausgleichsgraphen durch die gemessenen Punkte gelegt. Für die sinkenden Quanteneffizienzen ergeben sich dabei die in Abb. 11 dargestellten Graphen.

Die Graphen werden mit einem exponentiellem Fit der in Gl. (5) gezeigten Form modelliert.

$$
Q E=a * \exp \left(b^{*} d_{W}\right)
$$

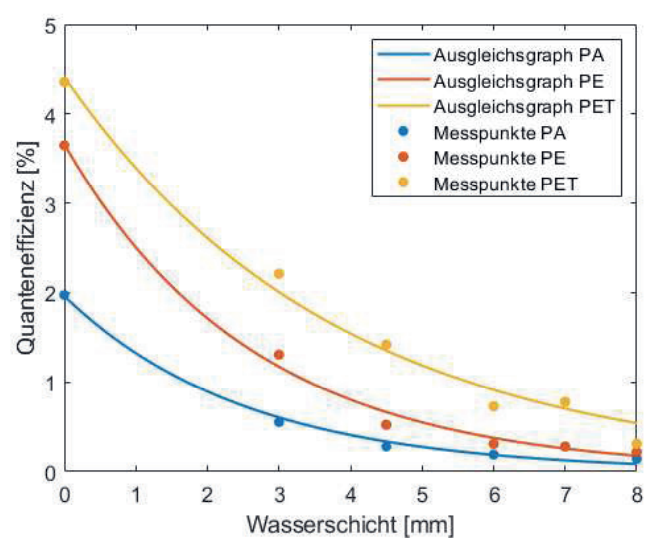

Abb. 11: Darstellung des Ausgleichsgraphen für die sinkende Quanteneffizienz der Kunststoffproben PA, PE und PET 
Tab. 3: Ermittelte Koeffizienten a und $b$ der exponentiellen Ausgleichgraphen für die Quanteneffizienz

\begin{tabular}{|c|c|c|}
\hline & $a[\%]$ & $b[1 / \mathrm{mm}]$ \\
\hline PA & 1,96 & $-0,39$ \\
\hline PE & 3,66 & $-0,38$ \\
\hline PET & 4,41 & $-0,27$ \\
\hline
\end{tabular}

Dabei ergeben sich für die Parameter $a$ und $b$ die in Tab. 3 eingetragenen Werte für die verschiedenen Kunststoffproben.

Der Koeffizient a gibt den kunststoffspezifischen Wert und $b$ den vom Wasser verursachten Verlust an.

Die gleiche Auswertung wird für die Apparatekonstante durchgeführt (siehe Abb. 12).

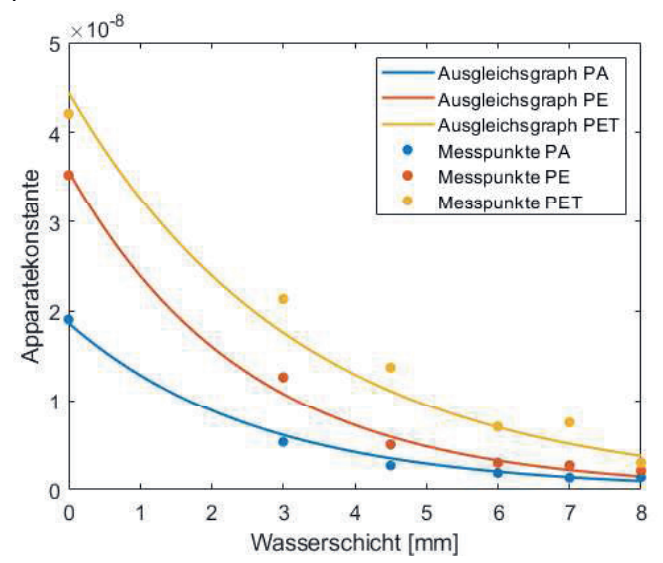

Abb. 12: Darstellung des Ausgleichsgraphen für die sinkende Apparatekonstante der Kunststoffproben PA, PE und PET

Die Koeffizienten für die Apparatekonstante sind in Tab. 4 eingetragen.

In beiden Fällen sind die Koeffizienten von $b$ erwartungsgemäß nahezu gleich, da sie die Absorbtionseigenschaften des Wassers darstellen.

Ein Vergleich der Werte für die ermittelten Quanteneffizienzen nach den Tab. 2 und 3 zeigen ebenfalls erwartungsgemäß keine signifikante Veränderung, da die Quanteneffizienz ein kunststoffspezifischer Wert ist, welcher nicht von Apparaten oder der Wasserschicht etc. abhängt.

Tab. 4: Ermittelte Koeffizienten a und $b$ der exponentiellen Ausgleichgraphen für die Apparatekonstante

\begin{tabular}{|c|c|c|}
\hline & $a[\%]$ & $b[1 / \mathrm{mm}]$ \\
\hline PA & $1,87 \cdot 10^{-8}$ & $-0,37$ \\
\hline PE & $3,57 \cdot 10^{-8}$ & $-0,40$ \\
\hline PET & $4,45 \cdot 10^{-8}$ & $-0,31$ \\
\hline
\end{tabular}

Quantitativ ergibt sich eine Veränderung des experimentell ermittelten Absorptionskoeffizienten $b$ im Vergleich zum Literaturwert aus [9]. Dieser ist um vier Größenordnungen niedriger als der Wert, der sich bei der experimentellen Auswertung zu ca. 0,35.1/mm ergibt.

Der Unterschied der beiden Werte ergibt sich daraus, dass der Absorptionskoeffizient des Referenzwertes aus [9] ohne den Einfluss von Streuung, Beugung und Brechung ermittelt worden ist. Im hier beschriebenen Aufbau sind diese Effekte enthalten und werden mit einem effektiven Absorptionskoeffizienten als Ersatzgröße berücksichtigt, die den Strahlungsverlust durch Absorption, Beugung, Streuung und Brechung von Wasser einschließt. Mit dieser Ersatzgröße gelingt die qualitative und quantitative Beschreibung der Experimente.

\section{Zusammenfassung der Ergebnisse}

Zusammenfassend betrachtet haben die simulativen und experimentellen Untersuchungen gezeigt, dass Kunststoffe in Wasser detektiert und identifiziert werden können.

Das aus [7, 8] bereits bekannte Verfahren wurde mit einem Absorptionskoeffizienten für Wasser so modifiziert, dass eine Erkennung und Charakterisierung des Kunststoffes in einer Wasserschicht ermöglicht wird.

Die Auswertung experimenteller Daten zeigt übereinstimmende Ergebnisse mit den in $[7,8]$ gewonnenen Quanteneffizienzen. Darüber hinaus wird ein exponentieller Abfall der Quanteneffizienz in Abhängigkeit der Wasserschichtdicke beobachtet. Der exponentielle Abfall wird qualitativ erwartet, jedoch ist der experimentell gewonnene Absorptionskoeffizient vier Größenordnungen größer ist als der Referenzwert aus [9]. Grund für die Abweichung ist, dass bei dem ReferenzAbsorptionskoeffizienten Effekte wie Streuung, Brechung und Beugung unberücksichtigt geblieben sind.

Für den exponentiellen Abfall wird eine Ersatzgröße gebildet, die diese Einflüsse berücksichtigt und in künftigen Untersuchungen statt des Literaturwertes verwendet wird.

Die Experimente legen nahe, dass eine Identifikation nur in geringen Wassertiefen möglich ist. Eine Detektion ohne Unterscheidung der Kunststoffe in Wasser ist hingegen auch in größeren Tiefen möglich.

Zukünftige Untersuchungen sollen zeigen, ob das Detektionsverfahren auch zum Nachweis von Mikroplastik in Wasser eingesetzt werden kann. 


\section{Literaturnachweis}

[1] Y. Tsang, C. Mak, C. Liebich, S. Lam, E. Sze, K. Chan, Microplastic pollution in the marine waters and sediments of Hong Kong, Marine Pollution Bulletin 115, 20-28 (2017): doi: 10.1016/j.marpolbul.2016.11.003.

[2] A. Karami, A. Golieskardi, Y. Ho, V. Larat, B. Salamatina, Microplastics in eviscerated flesh and excised organs of dried fish, Scientific Reports 7, 5473, 1-6 (2017); doi: 10.1038/s41598-017-05828-6

[3] S. Campbell, P. Williamson, B. Hall, Microplastics in the gastrointestinal tracts of fish and the water from an urban prairie creek, Facets Journal 2, 395-409 (2017); doi: 10.1139/facets-2017-0008

[4] A. Silva, A. Bastos, C. Justino, J. Costa, A. Duarte, T. Rocha-Santos, Microplastics in the environment: Challenges in analytical chemistry - A review, Analytica Chimica Acta 1017, 1-19 (2018); doi: 10.1016/j.aca.2018.02.043

[5] H. Langhals, D. Zgela, T. Schlücker, High Performance Recycling of Polymers by Means of Their Fluorescence Lifetimes, Green and Sustainable Chemistry 4, 144-150 (2014); doi: 10.4236/gsc.2014.43019

[6] H. Langhals, D. Zgela, T. Schlücker, Improved High Perfomance Recycling of Polymers by Means of Bi-Exponential Analysis of Their Fluorescence Lifetimes, Green and Sustainable Chemistry 5, 92-100 (2015); doi: 10.4236/gsc.2015.52012

[7] M. Wohlschläger, M. Versen, Bestimmung einer Quanteneffizienz für das automatisierte Sortieren von Kunststoffen durch Fluoreszenz, 16. AALE Konferenz, VDE Verlag Berlin, 1791872019 (2019); ISBN: 978-3-8007-4860-0

[8] M. Wohlschläger, M. Versen, H. Langhals: An apparatus specific quantum efficiency approach for sorting of plastics, IEEE Sensor Applications Symposium (SAS), Sophia Antipolis, 2019 (in print).

[9] G. Hale, R. Querry: Optical Constants of Water in the 200-nm to 200- $\mu \mathrm{m}$ Wavelength Region, Applied Optics12, 3, 555-563 (1973); doi: 10.1364/AO.12.000555

[10] Nichia, NVSU233B, Datasheet, 2018.

[11] Cascade Microtech, EPS150FA, A dedicated $150 \mathrm{~mm}$ manual probing solution for failure analysis, Datasheet, 2018

[12] Hamamatsu, Mini spectrometer $\mathrm{C10083CAH}$, Datasheet, 2018. 